\title{
Supertransvectants and symplectic geometry
}

\author{
H. Gargoubi $\ddagger \quad$ V. Ovsienko ${ }^{\ddagger}$
}

\begin{abstract}
The 1|1-supertransvectants are the osp(1|2)-invariant bilinear operations on weighted densities on the supercircle $S^{1 \mid 1}$, the projective version of $\mathbb{R}^{2 \mid 1}$. These operations are analogues of the famous Gordan transvectants (or Rankin-Cohen brackets). We prove that supertransvectants coincide with the iterated Poisson and ghost Poisson brackets on $\mathbb{R}^{2 \mid 1}$ and apply this result to construct star-products.
\end{abstract}

\section{Introduction}

\subsection{The transvectants and linear Poisson bracket: recapitulation}

Consider the space, denoted by $\mathcal{F}_{\lambda}$, of smooth (complex valued) functions on $S^{1}$ equipped with the following $\mathrm{SL}(2, \mathbb{R})$-action:

$$
f(x) \mapsto f\left(\frac{a x+b}{c x+d}\right)(c x+d)^{-2 \lambda}
$$

where $x$ is the affine coordinate and $\lambda \in \mathbb{C}$ is a parameter. Note that the space $\mathcal{F}_{\lambda}$ is naturally identified with the space of weighted densities of degree $\lambda$ ( $\lambda$-densities for short) via $\varphi=f(x)(d x)^{\lambda}$; the action (1.1) is then the standard action of fraction-linear coordinate transformations.

Classification of $\operatorname{SL}(2, \mathbb{R})$-invariant bilinear differential operators on $S^{1}$ acting in the spaces $\mathcal{F}_{\lambda}$ is a famous classical result of the invariant theory. For every $k=1,2,3, \ldots$, there exists the $\mathrm{SL}(2, \mathbb{R})$-invariant bilinear differential operator

$$
J_{k}^{\lambda, \mu}: \mathcal{F}_{\lambda} \otimes \mathcal{F}_{\mu} \rightarrow \mathcal{F}_{\lambda+\mu+k}
$$

given by the following explicit formula

$$
J_{k}^{\lambda, \mu}(f, g)=\sum_{i+j=k}(-1)^{i}\left(\begin{array}{c}
2 \lambda+k-1 \\
j
\end{array}\right)\left(\begin{array}{c}
2 \mu+k-1 \\
i
\end{array}\right) f^{(i)} g^{(j)},
$$

where $f^{(i)}(x)=\frac{d^{i} f(x)}{d x^{i}}$ and where $\left(\begin{array}{l}a \\ i\end{array}\right)=\frac{a(a-1) \cdots(a-i+1)}{i !}$. The operators (1.2), called transvectants, were found in 1885 by Gordan [7]; for almost all $(\lambda, \mu)$, these operators are unique $\mathrm{SL}(2, \mathbb{R})$ invariant bilinear differential operators on $S^{1}$ acting in the spaces $\mathcal{F}_{\lambda}$. Note that one can also assume $\lambda$ (half)integer and consider holomorphic functions on the upper half-plane $\mathcal{H}$.

\footnotetext{
${ }^{\ddagger}$ I.P.E.I.T., 2 Rue Jawaher Lel Nehru, Monfleury _ 1008 Tunis, TUNISIE; hichem.gargoubi@ipeit.rnu.tn,

${ }^{\S}$ CNRS, Institut Camille Jordan, Université Claude Bernard Lyon 1, 21 Avenue Claude Bernard, 69622 Villeurbanne Cedex, FRANCE; ovsienko@math.univ-lyon1.fr
} 
Transvectants have been rediscovered by Rankin [21] and Cohen [1] in the theory of modular forms and by Janson and Peetre [10] in differential projective geometry. Zagier [23] (see also [17]) noticed the coincidence between the Rankin-Cohen brackets and Gordan's transvectants. It was shown in [4] that the transvectants are in one-to-one correspondence with singular (i.e., vacuum or highest weight) vectors in the tensor product of two Verma modules over $\operatorname{sl}(2, \mathbb{C})$.

The best way to understand the operators (1.2) is, perhaps, to rewrite them in terms of the projective symplectic geometry, as in [19] and [20]. Consider the plane $\mathbb{R}^{2}$ with coordinates $(p, q)$ and the standard symplectic form $\omega=d p \wedge d q$ and the Poisson bracket

$$
\{F, G\}=\frac{\partial F}{\partial p} \frac{\partial G}{\partial q}-\frac{\partial F}{\partial q} \frac{\partial G}{\partial p} .
$$

The symmetry group of linear transformations in this case is the group $\operatorname{Sp}(2, \mathbb{R}) \simeq \operatorname{SL}(2, \mathbb{R})$. It is easy to describe all the $\operatorname{Sp}(2, \mathbb{R})$-invariant bilinear differential operators on $C^{\infty}\left(\mathbb{R}^{2}\right)$. For every positive integer $k$, there exists a bilinear differential operator of order $2 k$ given by the differential binomial of the form

$$
B_{k}(F, G):=\sum_{i+j=k}(-1)^{i}\left(\begin{array}{c}
k \\
i
\end{array}\right) \frac{\partial^{k} F}{\partial p^{i} \partial q^{j}} \frac{\partial^{k} G}{\partial p^{j} \partial q^{i}} .
$$

The operators $B_{k}$ are, in fact, iterations of the Poisson bracket in the following sense. Consider the operator $B$ on $C^{\infty}\left(\mathbb{R}^{2}\right) \otimes C^{\infty}\left(\mathbb{R}^{2}\right)$ given by

$$
B(F \otimes G)=\frac{\partial F}{\partial p} \otimes \frac{\partial G}{\partial q}-\frac{\partial F}{\partial q} \otimes \frac{\partial G}{\partial p}
$$

and the natural projection $\operatorname{Tr}(F \otimes G)=F G$. Then obviously

$$
B_{k}=\operatorname{Tr} \circ B^{k} .
$$

The expression (1.3) is, of course, much simpler than (1.2); in particular, it is independent of $\lambda$ and $\mu$. Nevertheless, these operators coincide up to a multiple. Identify the space $\mathcal{F}_{\lambda}\left(S^{1}\right)$ and the space of functions on $\mathbb{R}^{2} \backslash\{0\}$ homogeneous of degree $-2 \lambda$ by

$$
f(x) \longmapsto F_{f}(p, q)=p^{-2 \lambda} f\left(\frac{q}{p}\right),
$$

so that the affine coordinate is chosen as $x=q / p$.

Example 1.1. a) In the case $\lambda=-1$, the above formula identifies the 3 -dimensional space spanned by $\left\{1, x, x^{2}\right\}$ and the space of quadratic polynomials spanned by $\left\{p^{2}, p q, q^{2}\right\}$; this gives two realizations of $\mathrm{sl}(2)$ : in terms of vector fields on $S^{1}$ and Hamiltonian vector fields on $\mathbb{R}^{2}$, respectively.

b) In the case $\lambda=-\frac{1}{2}$, one identifies affine functions $1, x$ with linear functions $p, q$.

The following observation was made in [19].

Proposition 1.2. One has: $B_{k}\left(F_{f}, F_{g}\right)=k ! F_{J_{k}^{\lambda, \mu}(f, g)}$.

A simple corollary of Proposition 1.2 is the fact that the operators (1.2) can be used to construct an $\mathrm{SL}(2, \mathbb{R})$-invariant star-product on $T^{*} S^{1}$ (see [2], [19], [18] and [20]). Another application of the same idea leads to a multi-dimensional generalization of the transvectants as $\operatorname{Sp}(2 n, \mathbb{R})$-invariant bilinear differential operators on the sphere $S^{2 n-1}$, see [20]. Simple expression (1.3) allows one to avoid any non-trivial combinatorics. 
Remark 1.3. Formula (1.4) is somewhat mysterious, but it has a geometric sense. Every vector field on $S^{1}$ admits a unique "symplectic lift" to a homogeneous Hamiltonian vector field on $\mathbb{R}^{2} \backslash\{0\}$ and (1.4) is the unique lifting of weighted densities commuting with the vector fields lift (cf. [20]).

\subsection{The 1|1-supertransvectants}

We define the supercircle $S^{1 \mid 1}$ in terms of its superalgebra of functions: $C_{\mathbb{C}}^{\infty}\left(S^{1 \mid 1}\right)=C_{\mathbb{C}}^{\infty}\left(S^{1}\right)[\xi]$, where $\xi$ is an odd (Grassmann) coordinate, i.e., $\xi^{2}=0$ and $x \xi=\xi x$. In other words, this is the algebra of polynomials (of degree $\leq 1$ ) in $\xi$ with coefficients in $C_{\mathbb{C}}^{\infty}\left(S^{1}\right)$ :

$$
f(x, \xi)=f_{0}+\xi f_{1}
$$

where $f_{0}, f_{1}$ are smooth functions on $S^{1}$. The parity function $\sigma$ is defined on homogeneous in $\xi$ functions by setting $\sigma\left(f_{0}(x)\right)=0$ and $\sigma\left(\xi f_{1}(x)\right)=1$.

\section{The fractional-linear transformations}

The action of the supergroup $\operatorname{OSp}(1 \mid 2)$ on $S^{1 \mid 1}$ is given by the fraction-linear transformations

$$
(x, \xi) \mapsto\left(\frac{a x+b+\gamma \xi}{c x+d+\delta \xi}, \frac{\alpha x+\beta+e \xi}{c x+d+\delta \xi}\right),
$$

where $a d-b c-\alpha \beta=1, e^{2}+2 \gamma \delta=1, \alpha e=a \delta-c \gamma$ and $\beta e=b \delta-d \gamma$ (cf. [2, 16]).

We denote by $\mathcal{F}_{\lambda}$ the superspace of functions $C_{\mathbb{C}}^{\infty}\left(S^{1 \mid 1}\right)$ equipped with the following $\operatorname{OSp}(1 \mid 2)$ action

$$
f(x, \xi) \mapsto f\left(\frac{a x+b+\gamma \xi}{c x+d+\delta \xi}, \frac{\alpha x+\beta+e \xi}{c x+d+\delta \xi}\right)(c x+d+\delta \xi)^{-2 \lambda},
$$

where $\lambda \in \mathbb{C}$ is a parameter.

As usual, it is much easier to deal with the infinitesimal version of this action. The action of the orthosymplectic Lie superalgebra osp(1|2) on $S^{1 \mid 1}$ corresponding to the $\operatorname{OSp}(1 \mid 2)$-action is spanned by three even and two odd vector fields:

$$
\begin{aligned}
& \operatorname{osp}(1 \mid 2)_{0}=\operatorname{Span}\left(\frac{\partial}{\partial x}, \quad x \frac{\partial}{\partial x}+\frac{1}{2} \xi \frac{\partial}{\partial \xi}, \quad x^{2} \frac{\partial}{\partial x}+x \xi \frac{\partial}{\partial \xi}\right), \\
& \operatorname{osp}(1 \mid 2)_{1}=\operatorname{Span}(D, \quad x D),
\end{aligned}
$$

where

$$
D=\frac{\partial}{\partial \xi}+\xi \frac{\partial}{\partial x}
$$

is an odd vector field satisfying $\frac{1}{2}[D, D]=\frac{\partial}{\partial x}$.

The action of $\operatorname{osp}(1 \mid 2)$ on $\mathcal{F}_{\lambda}$ corresponding to the group action (1.5) is easy to calculate:

$$
\begin{aligned}
& L_{\frac{\partial}{\partial x}}^{\lambda}=\frac{\partial}{\partial x}, \quad L_{x \frac{\partial}{\partial x}}^{\lambda}=x \frac{\partial}{\partial x}+\lambda, \quad L_{x^{2} \frac{\partial}{\partial x}+x \xi \frac{\partial}{\partial \xi}}^{\lambda}=x^{2} \frac{\partial}{\partial x}+x \xi \frac{\partial}{\partial \xi},+2 \lambda x \\
& L_{D}^{\lambda}=D, \quad L_{x D}^{\lambda}=x D+2 \lambda \xi
\end{aligned}
$$

which is nothing but the Lie derivative of $\lambda$-densities (see, e.g., [3]).

Remark 1.4. Note that the odd elements $D$ and $x D$ generate the whole osp $(1 \mid 2)$ so that an operator commuting with the action of these two elements commutes with the OSp(1|2)-action. 
We will also use the following odd vector field on $S^{1 \mid 1}$

$$
\bar{D}=\frac{\partial}{\partial \xi}-\xi \frac{\partial}{\partial x}
$$

which defines the contact structure on $S^{1 \mid 1}$ since it spanns the kernel of the contact 1-form $\alpha=d x+\xi d \xi$, see [13, 3, 16] (Manin [15] calls this vector field the canonical SUSY-structure) 1 . It is characterized by the relations for the Lie superbrackets

$$
[D, \bar{D}]=0, \quad \frac{1}{2}[\bar{D}, \bar{D}]=-\frac{\partial}{\partial x} .
$$

An important property of $\bar{D}$ is that this vector field is invariant (up to multiplication by functions) under the $\operatorname{OSp}(1 \mid 2)$-action. In particular, one has $[x D, \bar{D}]=-\xi \bar{D}$.

Every differential operator on $S^{1 \mid 1}$ can be expressed in terms of $\bar{D}$. For instance, one has for the partial derivatives:

$$
\frac{\partial}{\partial x}=-\bar{D}^{2}, \quad \frac{\partial}{\partial \xi}=\bar{D}-\xi \bar{D}^{2}
$$

\subsection{Supertransvectants: an explicit formula}

The supertransvectants are the bilinear OSp(1|2)-invariant maps $J_{k}^{\lambda, \mu}: \mathcal{F}_{\lambda} \otimes \mathcal{F}_{\mu} \rightarrow \mathcal{F}_{\lambda+\mu+k}$ where $k=0, \frac{1}{2}, 1, \frac{3}{2}, 2, \ldots$. The supertransvectants were introduced by Gieres and Theisen in [6] and [5], see also [9]. Their (slightly modified) explicit formula is

$$
J_{k}^{\lambda, \mu}(f, g)=\sum_{i+j=2 k} C_{i, j}^{k} \bar{D}^{i}(f) \bar{D}^{j}(g),
$$

where the numeric coefficients are

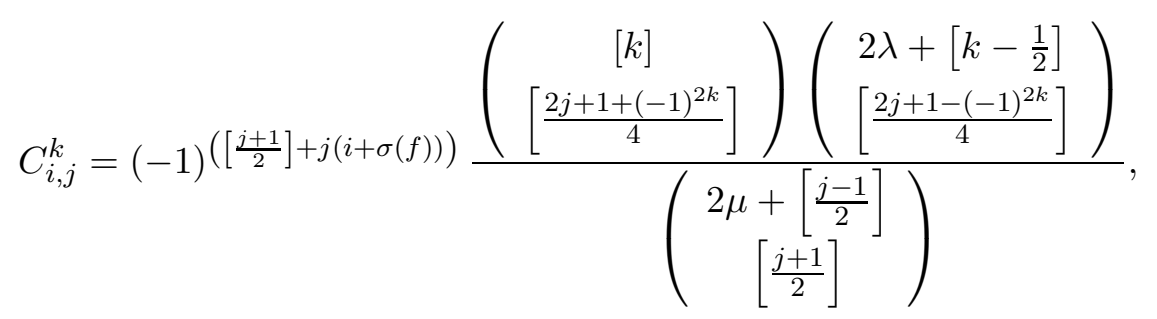

where $[a]$ denotes the integer part of $a \in \mathbb{R}$. It can be checked directly that these operators are, indeed, $\operatorname{OSp}(1 \mid 2)$-invariant.

\subsection{Comments}

It is an interesting feature of the supersymmetric case, that the operators labeled by integer $k$ are even, and by semi-integer $k$ are odd.

The two first examples of the supertransvectants, namely for $k=\frac{1}{2}$ and $k=1$, play a particular role. These operations are not only $\operatorname{OSp}(1 \mid 2)$-invariant, but also invariant with respect to the full infinite-dimensional conformal Lie superalgebra $\mathcal{K}(1)$ (also known as the centerless Neveu-Schwarz algebra); for a complete description of bilinear invariant $\mathcal{K}(N)$-operators for $n=1,2$ and 3 over contact vector fields with polynomial coefficients, see [14] and [13]. The

\footnotetext{
${ }^{1}$ For an invariant description of the operators $D$ and $\bar{D}$, in physical papers denoted by $Q$ and $D$, respectively, see 22 .
} 
first-order supertransvectant $J_{1}$ is nothing but the well-known contact bracket on $S^{1 \mid 1}$. The odd supertransvectant $J_{\frac{1}{2}}$ also belongs to the list of invariant operators from [14] and [13], but this operator is much less known. We will show that this operator defines a very interesting operation of "antibracket" on the $\mathcal{K}(1)$-modules of densities.

\subsection{The main results}

The main purpose of this paper is to give an interpretation of the supertransvectants in terms of the linear symplectic superspace $\mathbb{R}^{2 \mid 1}$ with coordinates $(p, q, \tau)$ and the standard symplectic form $\omega=d p \wedge d q+d \tau \wedge d \tau$. This interpretation considerably simplifies the explicit expression of the supertransvectants and their definition. It also allows one to apply some algebraic constructions of Poisson geometry, as star-products and suggests multi-dimensional generalizations of the supertransvectants.

The standard Poisson bracket on $\mathbb{R}^{2 \mid 1}$ is given by

$$
\{F, G\}=\frac{\partial F}{\partial p} \frac{\partial G}{\partial q}-\frac{\partial F}{\partial q} \frac{\partial G}{\partial p}+\frac{\partial F}{\partial \tau} \frac{\partial G}{\partial \tau} .
$$

Consider the space of functions on $\mathbb{R}^{2 \mid 1}$ with singularities at $(p, q)=(0,0)$ satisfying the condition $\mathcal{E}(F)=2 F$, where

$$
\mathcal{E}=p \frac{\partial}{\partial p}+q \frac{\partial}{\partial q}+\tau \frac{\partial}{\partial \tau}
$$

is the Euler field; such functions are called homogeneous of degree 2. This space is stable with respect to the bracket (1.9), therefore, it is a Lie (but not Poisson) superalgebra. This is nothing but the conformal superalgebra $\mathcal{K}(1)$.

We introduce one more, odd, operation on $C^{\infty}\left(\mathbb{R}^{2 \mid 1}\right)$ :

$$
\{F, G\}_{\mathrm{gPb}}=\frac{\partial F}{\partial \tau} \mathcal{E}(G)-(-1)^{\sigma(F)} \mathcal{E}(F) \frac{\partial G}{\partial \tau}+\tau\left(\frac{\partial F}{\partial p} \frac{\partial G}{\partial q}-\frac{\partial F}{\partial q} \frac{\partial G}{\partial p}\right),
$$

where $\sigma$ is the parity function. We call it the ghost Poisson bracket.

We will study the geometric and algebraic meaning of operation (1.10). Its crucial property is $\mathcal{K}(1)$-invariance.

Theorem 1. The ghost bracket (1.10) is invariant with respect to the action of the conformal algebra $\mathcal{K}(1)$.

It turns out that the Poisson bracket restricted to the homogeneous functions coincides with the supertransvectant $J_{1}$, while the ghost Poisson bracket coincides with $J_{\frac{1}{2}}$. In the framework of deformation quantization, we will consider "iterated" Poisson brackets (1.9) and (1.10).

Theorem 2. The supertransvectants $J_{k}$ with integer $k$ coincide with the iterated Poisson bracket (1.9), while those with semi-integer $k$ are obtained by the iteration of (1.9) with (1.10).

To the best of our knowledge, operations of type (1.10) have not been studied (see [12] for a survey of algebraic structures in Poisson geometry and [14 for that in supergeometry). Note that (1.10) is not invariant with respect to the full Poisson superalgebra $\left(C^{\infty}\left(\mathbb{R}^{2 \mid 1}\right),\{\},\right)$. 


\subsection{Open problems}

Grozman, Leites and Shchepochkina listed all simple Lie superalgebras of vector fields on the supercircles [8] (it is instructive to compare their list with that in [11]), and thus indicated the scope of work for possible superizations of Gordan's transvectants. The case we consider is the first on the agenda. Although there are four infinite series and several exceptional cases of simple stringy (or superconformal) superalgebras, there are only 7 (or, perhaps, 12: this has to be investigated) among them that contain the subalgebra of fraction linear transformations similar to the projective actions of $\operatorname{sl}(2)=\operatorname{sp}(2)$ or $\operatorname{osp}(1 \mid 2)$ considered here.

\section{The Poisson bracket and the ghost bracket}

Let us consider the first examples of supertransvectants: $J_{\frac{1}{2}}^{\lambda, \mu}$ and $J_{1}^{\lambda, \mu}$. To simplify the notations, throughout this section, we denote these operators by $($,$) and [, ], respectively.$

\subsection{The two operations}

The supertransvectant of order $\frac{1}{2}$ is

$$
(f, g)=\mu \bar{D}(f) g-(-1)^{\sigma(f)} \lambda f \bar{D}(g) .
$$

This odd operator is extremely interesting. We will show that it is invariant with respect to the full infinite-dimensional superconformal algebra (and therefore has a geometric meaning).

The first-order supertransvectant is

$$
[f, g]=\mu f^{\prime} g-\lambda f g^{\prime}-(-1)^{\sigma(f)} \frac{1}{2} \bar{D}(f) \bar{D}(g) .
$$

This even operation is nothing but the well-known Poisson bracket on $S^{1 \mid 1}$ (see, e.g., [13, [14] and also [3]).

\subsection{The Poisson superalgebra $\mathcal{F}$ and the conformal superalgebra $\mathcal{K}(1)$}

Consider the continuous sum (direct integral) of all spaces $\mathcal{F}_{\lambda}$ :

$$
\mathcal{F}=\cup_{\lambda \in \mathbb{C}} \mathcal{F}_{\lambda}
$$

the collection of operations $J_{1}^{\lambda, \mu}$ defines a bilinear map [, ]: $\mathcal{F} \otimes \mathcal{F} \rightarrow \mathcal{F}$.

Lemma 2.1. The operation $J_{1}$ defines the structure of a Poisson Lie superalgebra on $\mathcal{F}$.

Proof. Straightforward.

The space $\mathcal{F}_{-1} \subset \mathcal{F}$ is a Lie subalgebra since it is stable with respect to the bracket (2.2). This is precisely the conformal superalgebra on $S^{1 \mid 1}$, also known as the Lie superalgebra of contact vector fields (see [13, [8] and also [3]), or the (centerless) Neveu-Schwarz algebra. Let us denote this Lie subalgebra $\mathcal{K}(1)$. Each space $\mathcal{F}_{\lambda}$ is a $\mathcal{K}(1)$-module. 


\subsection{Invariance of the supertransvectant $J_{\frac{1}{2}}$}

The operation (2.1) is an additional, odd, bracket on the superspace $\mathcal{F}$. The crucial property of this ghost bracket is that it is invariant with respect to the action of the conformal subalgebra $\mathcal{K}(1) \subset \mathcal{F}$.

Proposition 2.2. The operation (2.1) on $\mathcal{F}$ is $\mathcal{K}(1)$-invariant.

Proof. One has to check that for $f \in \mathcal{F}_{-1}$ and arbitrary $g \in \mathcal{F}_{\mu}$ and $h \in \mathcal{F}_{\nu}$ one has

$$
[f,(g, h)]=(-1)^{\sigma(f)}([f, g], h)+(-1)^{\sigma(f)(\sigma(g)+1)}(g,[f, h]) .
$$

It can be done by straightforward calculation. Note however, that the identity (2.3) is a particular case of Theorem 1 whose proof will be given in Section 3.2.

\subsection{The algebraic structure on $\mathcal{F}_{-\frac{1}{2}}$}

The $\mathcal{K}(1)$-module $\mathcal{F}_{-\frac{1}{2}}$ is a "square root" of $\mathcal{K}(1) \cong \mathcal{F}_{-1}$. This space is stable with respect to the operation (, ). Adopting the basis

$$
V_{n}=x^{n+\frac{1}{2}}, \quad \Psi_{n}=\xi x^{n},
$$

one obtains explicitly

$$
\begin{aligned}
\left(V_{n}, V_{m}\right) & =(m-n) \Psi_{n+m}, \\
\left(\Psi_{n}, V_{m}\right) & =V_{n+m}=-\left(V_{m}, \Psi_{n}\right), \\
\left(\Psi_{n}, \Psi_{m}\right) & =2 \Psi_{n+m} .
\end{aligned}
$$

Proposition 2.3. The algebra $\left(\mathcal{F}_{-\frac{1}{2}},(),\right)$ satisfies the following four properties:

1. the odd part $\left(\mathcal{F}_{-\frac{1}{2}}\right)_{1}$ is a commutative associative subalgebra;

2. the odd part $\left(\mathcal{F}_{-\frac{1}{2}}\right)_{1}$ acts on the even part $\left(\mathcal{F}_{-\frac{1}{2}}\right)_{0}$ by $\rho_{\psi} v:=(\psi, v)$ and one has

$$
\rho_{\varphi} \circ \rho_{\psi}+\rho_{\psi} \circ \rho_{\varphi}=\rho_{(\varphi, \psi)}
$$

for all $\varphi, \psi \in\left(\mathcal{F}_{-\frac{1}{2}}\right)_{1}$;

3. the map $():,\left(\mathcal{F}_{-\frac{1}{2}}\right)_{0} \otimes\left(\mathcal{F}_{-\frac{1}{2}}\right)_{0} \rightarrow\left(\mathcal{F}_{-\frac{1}{2}}\right)_{1}$ is anti-symmetric and $\left(\mathcal{F}_{-\frac{1}{2}}\right)_{1}$-invariant, namely

$$
\rho_{\psi}(v, w)=\left(\rho_{\psi} v, w\right)+\left(v, \rho_{\psi} w\right)
$$

for all $\psi \in\left(\mathcal{F}_{-\frac{1}{2}}\right)_{1}$ and $v, w \in\left(\mathcal{F}_{-\frac{1}{2}}\right)_{0}$;

4. the Jacobi identity is satisfied:

$$
(u,(v, w))+(v,(w, u))+(w,(u, v))=0,
$$

for all $u, v, w \in\left(\mathcal{F}_{-\frac{1}{2}}\right)_{0}$.

Proof. The properties 1-4 of can be checked directly. 


\section{$3 \quad$ The symplectic lifting}

In this section we show that the supertransvectants (2.2) and (2.1) coincide with the Poisson bracket (1.9) and the ghost bracket (1.10). We prove Theorem 1

\subsection{Homogeneous functions}

Let us define a symplectic lifting of the space $\mathcal{F}$. To any function $f \in \mathcal{F}_{\lambda}$ we associate a function on $\mathbb{R}^{2 \mid 1}$ homogeneous of degree $-2 \lambda$. The explicit formula is $f(x, \xi) \mapsto F_{f}(p, q, \tau)$, where

$$
F_{f}(p, q, \tau)=p^{-2 \lambda} f\left(\frac{q}{p}, \frac{\tau}{p}\right) \equiv p^{-2 \lambda} f_{0}\left(\frac{q}{p}\right)+\tau p^{-2 \lambda-1} f_{1}\left(\frac{q}{p}\right)
$$

and where $(p, q, \tau)$ are coordinates on $\mathbb{R}^{2 \mid 1}$. Abusing the notations, from now on, we will also denote $\mathcal{F}_{\lambda}$ the space of homogeneous functions on $\mathbb{R}^{2 \mid 1}$ of degree $-2 \lambda$.

This lifting is invariant in the following sense.

Proposition 3.1. (i) The 1-transvectant $J_{1}^{\lambda, \mu}$, see the explicit formula (2.2), corresponds to the Poisson bracket (1.9):

$$
F_{[f, g]}=\frac{1}{2}\left\{F_{f}, F_{g}\right\},
$$

(ii) The $\frac{1}{2}$-transvectant (2.1) corresponds to the odd bracket (1.10):

$$
F_{(f, g)}=-\frac{1}{2}\left\{F_{f}, F_{g}\right\}_{\mathrm{gPb}}
$$

Proof. Just substitute the expression (3.1) to (1.9) and (1.10) and compare the result with (2.2) and (2.1).

A nice feature of the lifting (3.1) is that it intertwines the standard embedding of osp $(1 \mid 2)$ into the Poisson algebra given by the quadratic polynomials

$$
\operatorname{osp}(1 \mid 2)=\operatorname{Span}\left(p^{2}, p q, q^{2} ; \quad \tau p, \tau q\right)
$$

with the $\operatorname{osp}(1 \mid 2)$-action (1.6). Again, the odd elements $\tau p$, and $\tau q$ generate the whole algebra.

Remark 3.2. The lifting (3.1) has a similar geometric meaning as that of (1.4). The Lie superalgebra $\mathcal{K}(1) \cong \mathcal{F}_{-1}$ corresponds to the space of functions on $\mathbb{R}^{2 \mid 1}$ homogeneous of degree 2 and formula (3.1) is the unique way to identify weighted densities with homogeneous functions that intertwines (2.2) and (1.9).

\subsection{Invariance of the ghost Poisson bracket}

Let us prove Theorem 1 .

To show that the ghost bracket (1.10) is invariant with respect to the action of $\mathcal{K}(1)$, one has to show that

$$
\left\{F,\{G, H\}_{\mathrm{gPb}}\right\}=(-1)^{\sigma(F)}\{\{F, G\}, H\}_{\mathrm{gPb}}+(-1)^{\sigma(F)(\sigma(G)+1)}\{G,\{F, H\}\}_{\mathrm{gPb}}
$$

for every function $F \in \mathcal{F}_{-1}$. To do this, we adopt the technique routine in Poisson geometry. The bracket (1.10) is given by the following "ghost Poisson" bivector

$$
\Lambda=\frac{\partial}{\partial \tau} \wedge \mathcal{E}+\tau P
$$


where $P=\frac{\partial}{\partial p} \wedge \frac{\partial}{\partial q}$ is the even part of the Poisson bivector. The equivariance condition is equivalent to the fact that the Hamiltonian vector field, $X_{F}$, with respect to the Poisson bracket (1.9) preserves the bivector $\Lambda$ that can be readily checked.

Remark 3.3. There is a uniqueness statement. It follows from the classification of the supertransvectants, that, for generic $(\lambda, \mu)$, the ghost bracket (1.10) is a unique odd bilinear homogeneous map $\mathcal{F}_{\lambda} \otimes \mathcal{F}_{\mu} \rightarrow \mathcal{F}_{\nu}$ commuting with the $\mathcal{K}(1)$-action.

\section{Supertransvectants from the symplectic viewpoint}

In this section we prove Theorem 2, We realize the supertransvectants in terms of the iterated brackets (1.9) and (1.10). As a corollary of this result, we construct a star-product involving the supertransvectants $J_{k}$ as $k$-th order terms.

\subsection{Even supertransvectants as the iterated Poisson bracket}

Consider the linear operator $\mathcal{B}$ acting on the space $C^{\infty}\left(\mathbb{R}^{2 \mid 1}\right) \otimes C^{\infty}\left(\mathbb{R}^{2 \mid 1}\right)$ given by

$$
\mathcal{B}(F \otimes G)=\frac{\partial F}{\partial p} \otimes \frac{\partial G}{\partial q}-\frac{\partial F}{\partial q} \otimes \frac{\partial G}{\partial p}+\frac{\partial F}{\partial \tau} \otimes \frac{\partial G}{\partial \tau}
$$

The Poisson bracket (1.9) is given by the composition: $\{\}=,\operatorname{Tr} \circ \mathcal{B}$ where $\operatorname{Tr}$ is the operator of projection $\operatorname{Tr}(F \otimes G)=F G$.

Define the "iterated Poisson brackets" $\mathcal{B}_{k}=\operatorname{Tr} \circ \mathcal{B}^{k}$, with $k=1,2, \ldots$ One readily gets the explicit formula:

$$
\mathcal{B}_{k}(F, G)=B_{k}(F, G)+k B_{k-1}\left(\frac{\partial F}{\partial \tau}, \frac{\partial G}{\partial \tau}\right),
$$

where $B_{k}$ is the iterated bracket (1.3) on $\mathbb{R}^{2}$.

Proposition 4.1. The iterated Poisson bracket (4.2) is osp(1|2)-invariant for every integer $k$.

Proof. The osp(1|2)-action on $\mathbb{R}^{2 \mid 1}$ is generated by two odd elements: $\tau p$ and $\tau q$. Let us check that

$$
\left\{t p, \mathcal{B}_{k}(F, G)\right\}=\mathcal{B}_{k}(\{t p, F\}, G)+(-1)^{\sigma(F)} \mathcal{B}_{k}(F,\{t p, G\}) .
$$

If $F, G$ are even then the above relation is evident. For $F$ even and $G=\tau G_{1}$ odd one has the condition

$$
p B_{k}\left(F, G_{1}\right)=k B_{k-1}\left(\frac{\partial F}{\partial q}, G_{1}\right)+B_{k}\left(F, p G_{1}\right)
$$

that follows from formula (1.3). Finally, for $F=\tau F_{1}, G=\tau G_{1}$, one gets the relation:

$$
k \tau\left(B_{k-1}\left(F_{1}, G_{1}\right)\right)_{q}=\tau\left(B_{k}\left(p F_{1}, G_{1}\right)-B_{k}\left(F_{1}, p G_{1}\right)\right)
$$

which is obviously true.

The bilinear map $\mathcal{B}_{k}$ restricted to the homogeneous functions defines the map

$$
\mathcal{B}_{k}: \mathcal{F}_{\lambda} \otimes \mathcal{F}_{\mu} \rightarrow \mathcal{F}_{\lambda+\mu+k}
$$

which is osp $(1 \mid 2)$-invariant. It follows then from the uniqueness of the supertransvectants that the maps $J_{k}^{\lambda, \mu}$ and $\left.\mathcal{B}_{k}\right|_{\mathcal{F}_{\lambda} \otimes \mathcal{F}_{\mu}}$ are proportional. Taking particular functions $p^{-2 \lambda}$ and $q^{-2 \mu}$, one now checks that the proportionality coefficient is $2^{k} k$ ! and finally

$$
F_{J_{k}^{\lambda, \mu}(f, g)}=\frac{1}{2^{k} k !} \mathcal{B}_{k}\left(F_{f}, F_{g}\right) .
$$

for generic, and therefore, for all $(\lambda, \mu)$. 


\subsection{Iterated ghost Poisson bracket and the odd supertransvectants}

Define an analogous linear operator corresponding to the ghost bracket (1.10) by the following formula:

$$
\begin{aligned}
\partial(F \otimes G)= & \frac{\partial F}{\partial \tau} \otimes \mathcal{E}(G)-(-1)^{\sigma(F)} \mathcal{E}(F) \otimes \frac{\partial G}{\partial \tau} \\
& +\chi(f, g)\left(\tau \frac{\partial F}{\partial p} \otimes \frac{\partial G}{\partial q}-\tau \frac{\partial F}{\partial q} \otimes \frac{\partial G}{\partial p}+\frac{\partial F}{\partial p} \otimes \tau \frac{\partial G}{\partial q}-\frac{\partial F}{\partial q} \otimes \tau \frac{\partial G}{\partial p}\right)
\end{aligned}
$$

where $\chi(f, g)$ is a function depending on the parity of $f$ and $g$ :

$$
\chi(f, g)=\frac{1}{2}+\frac{\left(1+(-1)^{(\sigma(f)+1)(\sigma(g)+1)}\right)}{4}
$$

Clearly

$$
\{,\}_{\mathrm{gPb}}=\operatorname{Tr} \circ \partial .
$$

Let us define the odd iterated brackets:

$$
\mathcal{B}_{k+\frac{1}{2}}=\operatorname{Tr} \circ \partial \circ \mathcal{B}^{k}
$$

for $k=1,2, \ldots$.

Proposition 4.2. The odd brackets $\mathcal{B}_{k+\frac{1}{2}}$ are osp(1|2)-invariant.

Proof. Similar to the proof of Proposition 4.1 .

Again, the proportionality coefficient can be calculated:

$$
F_{J_{k+\frac{1}{2}}^{\lambda, \mu}(f, g)}=-\frac{1}{2^{k} k !} \mathcal{B}_{k+\frac{1}{2}}\left(F_{f}, F_{g}\right)
$$

Remark 4.3. (i) The definition (4.5) does not depend on the order of composition of the operators $\partial$ and $\mathcal{B}$ since one has

$$
\operatorname{Tr} \circ \partial \circ \mathcal{B}^{k}=\operatorname{Tr} \circ \mathcal{B}^{\ell} \circ \partial \circ \mathcal{B}^{m}
$$

for $\ell+m=k$.

(ii) the map (4.4) is the "square root" of the map (4.1) in the following sense:

$$
\operatorname{Tr} \circ \partial^{2}=\frac{1}{2}\left(\left(1+(-1)^{\sigma(F)(\sigma(G)+1)}\right)(\mu+1)-\left(1+(-1)^{\sigma(G)(\sigma(F)+1)}\right)(\lambda+1)\right) \operatorname{Tr} \circ \mathcal{B},
$$

when restricted to the homogeneous functions $\mathcal{F}_{\lambda} \otimes \mathcal{F}_{\mu}$.

\subsection{An $\operatorname{osp}(1 \mid 2)$-invariant star-product}

The coincidence (4.3) defines a pull-back of the standard Moyal-Weyl star-product on $\mathbb{R}^{2 \mid 1}$ to an invariant star-product on the Poisson algebra $\mathcal{F}$. The explicit formula is very simple:

$$
f * g=f g+\sum_{k=1}^{\infty} t^{k} J_{k}^{\lambda, \mu}(f, g),
$$

for all $f \in \mathcal{F}_{\lambda}$ and $g \in \mathcal{F}_{\mu}$. The operation (4.7) is an associative product on the space of formal series $\mathcal{F}[[t]]$ which is a deformation of the standard commutative product of functions. The star-product (4.7) is obviously osp $(1 \mid 2)$-invariant.

Note that the operation (4.7) involves only even supertransvectants. It would be interesting to understand if there is another deformation that contains the odd terms as well. 


\section{Appendix}

For the sake of completeness, let us give here a proof of the fact that, for the generic $(\lambda, \mu)$, the supertransvectants (1.7) with coefficients (1.8) are the unique osp(1|2)-invariant bidifferential operators.

An arbitrary bidifferential operator can be written in the form (1.7) with coefficients $C_{i, j} \in$ $C^{\infty}\left(S^{1 \mid 1}\right)$. The action of a vector field $X$ on the operator (1.7) is then given by

$$
\mathcal{L}(B)(f, g):=\sum_{i+j=k} C_{i, j}\left(L_{X}\left(\bar{D}^{i}\right)(f) \bar{D}^{j}(g)+(-1)^{i+\sigma(f)} \bar{D}^{i}(f) L_{X}\left(\bar{D}^{j}\right)(g)\right)
$$

We will use the generators $D$ and $x D$ of osp $(1 \mid 2)$. The invariance condition with respect to the first generator $D$ proves that each $C_{i, j}$ is an even constant. Consider the vector field $x D$. First, we calculate the action of $x D$ on the operators $\bar{D}^{i}: \mathcal{F}_{\lambda} \rightarrow \mathcal{F}_{\mu}$. One has

$$
\begin{aligned}
L_{x D}\left(\bar{D}^{2 p+1}\right) & :=(x D+2 \mu \xi) \bar{D}^{2 p+1}+\bar{D}^{2 p+1}(x D+2 \lambda \xi) \\
& =(2 \lambda+p) \bar{D}^{2 p}+(2 \mu-2 \lambda-2 p-1) \xi \bar{D}^{2 p+1}
\end{aligned}
$$

for $i=2 p+1$ and

$$
\begin{aligned}
L_{x D}\left(\bar{D}^{2 p}\right) & :=(x D+2 \mu \xi) \bar{D}^{2 p}-\bar{D}^{2 p}(x D+2 \lambda \xi) \\
& =p \bar{D}^{2 p-1}+(2 \mu-2 \lambda-2 p) \xi \bar{D}^{2 p}
\end{aligned}
$$

for $i=2 p$. In particular, if $\mu=\lambda+\frac{i}{2}$, one obtains

$$
L_{x D}^{\lambda, \lambda+\frac{i}{2}}\left(\bar{D}^{i}\right)= \begin{cases}\left(2 \lambda+\frac{(i-1)}{2}\right) \bar{D}^{i-1} & \text { if } i \text { is odd } \\ \frac{i}{2} \bar{D}^{i-1} & \text { if } i \text { is even }\end{cases}
$$

The equivariance equation, $L_{x D}(J)(f, g)=0$, for a bidifferential operator $J$ gives now the following system:

$$
\begin{aligned}
(2 \lambda+l) C_{2 l+1,2 m} & =-(-1)^{\sigma(f)}(2 \mu+m) C_{2 l, 2 m+1} \\
l C_{2 l, 2 m-1} & =(-1)^{\sigma(f)} m C_{2 l-1,2 m} \\
(2 \lambda+l) C_{2 l+1,2 m-1} & =-(-1)^{\sigma(f)} m C_{2 l, 2 m} \\
l C_{2 l, 2 m} & =(-1)^{\sigma(f)}(2 \mu+m) C_{2 l-1,2 m+1}
\end{aligned}
$$

Explicit solution of the system (5.1) leads to the following critical (or "resonant") set

$$
I_{k}=\left\{0,-\frac{1}{2},-1,-\frac{3}{2} \ldots,-\frac{1}{2}\left[\frac{k-1}{2}\right]\right\}
$$

and one has to separate the following four cases.

1) If $\lambda, \mu \notin I_{k}$, then the system (5.1) has a unique (up to a multiplicative constant) solution given by (1.8).

2) If one of the weights $\lambda$ or $\mu$ belongs to $I_{k}$ but the second one does not, then the system (5.1) has a unique (up to a multiplicative constant) solution. If, say, $\lambda=\frac{1-m}{4}$ for some odd $m$, then the corresponding bilinear osp(1|2)-invariant operator is given by

$$
f \otimes g \longmapsto J_{k-m}^{\frac{1+m}{4}, \mu}\left(\bar{D}^{m}(f), g\right) .
$$


3) If $\lambda=\frac{1-m}{4}$ for some odd $m$ and $\mu=\frac{1-\ell}{4}$ for some odd $\ell$, and if $\ell+m>k$, then the solution is still unique and is of the form

$$
f \otimes g \longmapsto J_{k-m}^{\frac{1+m}{4}, \mu}\left(\bar{D}^{m}(f), g\right)=J_{k-\ell}^{\lambda, \frac{1+\ell}{4}}\left(f, \bar{D}^{\ell}(g)\right) .
$$

4) If $\lambda=\frac{1-m}{4}$ for some odd $m$ and $\mu=\frac{1-\ell}{4}$ for some odd $\ell$, and if $\ell+m>k$, then there are two independent solutions

$$
f \otimes g \longmapsto J_{k-m}^{\frac{1+m}{4}, \mu}\left(\bar{D}^{m}(f), g\right)=J_{k-\ell}^{\lambda, \frac{1+\ell}{4}}\left(f, \bar{D}^{\ell}(g)\right) .
$$

and

$$
f \otimes g \longmapsto J_{k-m-\ell}^{\frac{1+m}{4}, \frac{1+\ell}{4}}\left(\bar{D}^{m}(f), \bar{D}^{\ell}(g)\right)
$$

Acknowledgements. It is a great pleasure to thank Christian Duval and Dimitry Leites for numerous enlightening discussions and help. We are also grateful to Charles Conley, François Gieres, Claude Roger and Serge Parmentier for their valuable comments at different stages of this work.

\section{References}

[1] H. Cohen, Sums involving the values at negative integers of L-functions of quadratic characters, Math. Ann. 217 (1975) 271-285.

[2] P. Cohen, Yu. Manin and D. Zagier, Automorphic pseudodifferential operators, Algebraic aspects of integrable systems, 17-47, Progr. Nonlinear Differential Equations Appl., 26, Birkhäuser Boston, Boston, MA, 1997.

[3] H. Gargoubi, N. Mellouli, V. Ovsienko, Differential operators on supercircle: conformally equivariant quantization and symbol calculus, Lett. Math. Phys., 79 (2007) 51-65.

[4] A.M. El Gradechi, The Lie theory of the Rankin-Cohen brackets and allied bi-differential operators, Adv. in Math. 207 (2006) 484-531.

[5] F. Gieres, Conformally covariant operators on Riemann surfaces (with application to conformal and integrable models), Int. J. Mod. Phys. A, 8 (1993) 1-58.

[6] F. Gieres, S. Theisen, Superconformally covariant operators and super W-algebras, J. Math. Phys. 34 (1993) 5964-5985.

[7] P. Gordan, Invariantentheorie, Teubner, Leipzig, 1887.

[8] Grozman P., Leites D., Shchepochkina I., Lie superalgebras of string theories., Acta Mathematica Vietnamica, v. 26, 2001, no. 1, 27-63; hep-th/9702120

[9] W-J. Huang, Superconformal covariantization of superdifferential operator on (1|1) superspace and classical $N=2 W$ superalgebras, J. Math. Phys. 35:5 (1994) 2570-2582.

[10] S. Janson, J. Peetre, A new generalization of Hankel operators(the case of higher weights), Math. Nachr. 132 (1987) 313-328. 
[11] V.G. Kac, Classification of supersymmetries, Proc. Int. Congress of Math., Vol. I (Beijing, 2002), 319-344, Higher Ed. Press, Beijing, 2002.

[12] Y. Kosmann-Schwarzbach, Derived brackets, Lett. Math. Phys. 69 (2004), 61-87.

[13] D. Leites, Lie superalgebras, Current problems in mathematics, 25, 3-49, Akad. Sci. SSSR, Moscow, 1984.

[14] Leites D., Kochetkov Yu., Weintrob A., New invariant differential operators on supermanifolds and pseudo-(co)homology, Lecture Notes in Pure and Appl. Math., 134, Dekker, N.Y., 1991, 217-238.

[15] Yu.I. Manin, Topics in Non-commutative geometry, Princeton Univ. Press, Princeton, 1979.

[16] J.-P. Michel, C. Duval, On the projective geometry of the supercircle: a unified construction of the super cross-ratio and Schwarzian derivative, math-ph/0710.1544.

[17] P.J. Olver, J.A. Sanders, Transvectants, modular forms and the Heisenberg algebra, Adv. in Appl. Math. 25 (2000) 252-283.

[18] H. Omori, Y. Maeda, N. Miyazaki, A. Yoshioka, Deformation quantization of the poisson algebra of Laurent polynomials, Lett. Math. Phys. 46 (1998) 171-180.

[19] V. Ovsienko, Exotic deformation quantization, J. Differential Geom. 45 (1997) 390-406.

[20] Ovsienko V., Tabachnikov S., Projective differential geometry old and new: from the Schwarzian derivative to cohomology of diffeomorphism groups, Cambridge University Press, 2005.

[21] R.A. Rankin, The construction of automorphic forms from the derivatives of a given form, J. Indian Math. Soc. 20 (1956), 103-116.

[22] Shchepochkina I., How to realize Lie algebras by vector fields, Theor. Math. Phys. 147:3, 2006, 821-838; math.RT/0509472.

[23] D. Zagier, Modular forms and differential operators, Proc. Indian Acad. Sci. (Math. Sci.) 104 (1994), 57-75. 